\title{
INTRACAPSULAR EXTRACTION OF INTUMESCENT CATARACT BY APPLICATION OF LOW TEMPERATURE*
}

BY

\section{T. KRWAWICZ}

Ophthalmological Clinic, Medical Academy, Lublin, Poland

THE technical improvements recently introduced into cataract surgery, and especially the application of enzymatic zonulolysis, promise to make the extraction of the cataractous lens easier and safer. There remain, however, some potential sources of difficulty, and no procedure is free from the risk of unexpected and often dangerous complications. Enzymatic abolition of the resistance of the zonule fibres, or their complete breaking, are not the only essential conditions for a smooth intracapsular cataract extraction; the condition of the lens capsule is just as important. When the capsule is thinned because of degenerative changes, or excessively tightened, the proposed intracapsular extraction may fail, even if the resistance of the zonule fibres has been abolished. Other complications may result from unreliable methods of grasping the capsule.

Writers who deal with the results of cataract extraction aided by zonulolysis have occasionally reported the rupture of the lens capsule (Barraquer, 1959; Orłowski, 1959). This means that zonulolysis, the usefulness of which has not yet been finally proved, will not settle the problem as long as the technique of grasping the capsule remains imperfect. It seems therefore reasonable to concentrate on the other essential factor in an uncomplicated intracapsular cataract extraction, i.e. the method by which the lens capsule is grasped.

It is generally agreed that an intumescent cataract should be extracted by means of the erisophake, but it can often be extracted quite as easily with the forceps, especially when the capsule is thickened by senile proliferation. In other cases micropuncture may be helpful, or the cataractous lens may be tumbled by the method of Smith (1926) and Hruby (1957). The use of diathermy, as suggested by Lacarrère (1932) and Jess (1931), which is of value in extracting luxated lenses, is less suitable for the extraction of intumescent cataract. None of these methods, however, even if combined with zonulolysis, eliminates completely the danger of the rupture of the lens capsule.

The present paper reports a method of extracting intumescent cataract, which eliminates, or at least greatly reduces, this difficulty, without regard to the tension or friability of the lens capsule. 


\section{Method}

The name "cryoextractor" is suggested for the pencil-shaped, ball-tipped instrument which has been evolved for this operation. It is made of copper (which is an excellent conductor of heat) and nickelplated (Fig. 1). Before use it is placed in a thermos flask containing a mixture of dry ice and methyl alcohol, and it quickly assumes the temperature of its environment, i.e. about $-79^{\circ} \mathrm{C}$. To assure asepsis, the sterile cryoextractor is not placed directly in the freezing mixture, but in a small silver cylinder filled with ethyl alcohol. If required, it can be placed in a deep-freeze provided with an alcohol thermostat. The copper head is mounted in a plastic holder, which facilitates manipulation and protects the surgeon's hands against the low temperature. A cotton glove should also be used as an additional protection. Before application, the tip is wiped with a sterile napkin to remove the remaining alcohol.

When the lens capsule is touched with the tip of the instrument, an immediate local freezing occurs of the capsule and the subjacent part of the cataractous

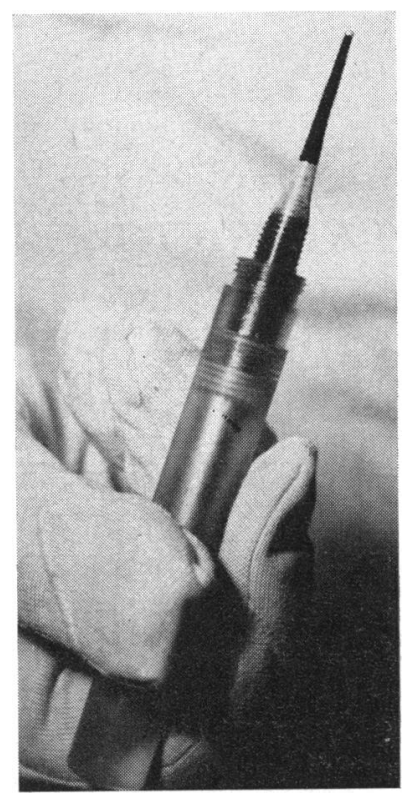

Fig. 1.-Cryoextractor. mass, which attach themselves to the cold metal, so firmly as to ensure an easy breaking of the zonule fibres and extraction of the lens.

Our experiments on extracted human lenses and on animals have shown that the partial refrigeration of the lens occurs more quickly if the tip of the cryoextractor is dipped into water so that a thin coating of ice is formed on the metal before it is applied to the lens. Although the refrigeration is limited to a relatively small part of the lens, it is sufficient to withstand the manipulations which are necessary to break the zonule fibres of the cadaver eye.

Some of our experiments were intended to investigate the possibility of complications. It appears that partial refrigeration of the lens has no harmful effect on the other parts of the animal eye. When the cornea is touched it immediately and firmly adheres to the metal but no permanent injury is inflicted on the cornea if the contact between it and the instrument is promptly interrupted with a spatula. In a similar way, the adhering iris can be freed from the cryoextractor by sliding a spatula along its wall. In all cases prompt action prevents further complications.

These complications may be prevented by insulating the tapering end of the cryoextractor with a silk thread, only the ball-shaped tip being left bare, so that direct contact between the surrounding ocular tissues and the cold metal becomes very improbable.

\section{Operative Technique}

The patient is prepared in the usual way. The corneo-scleral suture described by Liégard (1913) is laid with special care; the bite should take in half the thickness of the cornea so that the suture can be used for lifting the 
corneal flap to expose the lens. The iris is incised at 11 and 1 o'clock and pulled upwards with a retractor or forceps to ensure better access to the anterior surface of the lens. Pressure is exerted on the lower part of the cornea, so that the upper part of the lens inclines towards the operative wound. It is then that the ice-coated tip of the cryoextractor is applied to the lens capsule near its equator at 12 o'clock. This is immediately followed by a freezing of the capsule and subcapsular masses over an area slightly larger than that of the contact between the capsule and the tip of the cryoextractor. The zonule fibres are broken with rotating movements to the right and to the left, and the lens is then delivered by sliding (Figs 2 to 6 , overleaf).

As the instrument is insulated with silk, except for its ball-shaped end, neither the cornea nor the iris will stick to it if inadvertently touched. When the insulation becomes hoar-frosted, the risk of catching the cornea or the iris increases, but by this time the extraction of the lens should be far enough advanced to make such a complication extremely unlikely.

\section{Results}

Fifty extractions of intumescent cataracts have been performed by this method. In 48 cases the cataract could be extracted intracapsularly. In only two cases did the capsule rupture near the lower part of the equator during the final stage of the operation, and in both cases it was possible to remove the capsule completely thanks to its firm attachment to the cryoextractor. The cataractous masses were easily removed from the anterior chamber or from the operative wound, but in one case some lens debris remained under the iris, perhaps because excessive shrinking of the thinned capsule where the instrument was applied produced a rupture of the capsule at the opposite point. This complication could probably be avoided by delaying the application of the cryoextractor while it has lost some of its freezing power. In one case there was some vitreous loss, which occurred when the corneo-scleral suture was being tied, and in two cases choroidal detachment was observed at a later stage.

In 49 cases full visual acuity was obtained, and in the case in which some lens debris remained under the iris the visual acuity was 5/10.

\section{Conclusions}

The clinical observations presented above show that our method of grasping the lens capsule gives better protection against the rupture of the capsule than any of the current methods. This is mainly due to the fact that, thanks to the refrigeration and solidification of the subcapsular masses, some part of the traction exerted during the operation goes beyond the capsule, reducing the risk of rupture. 


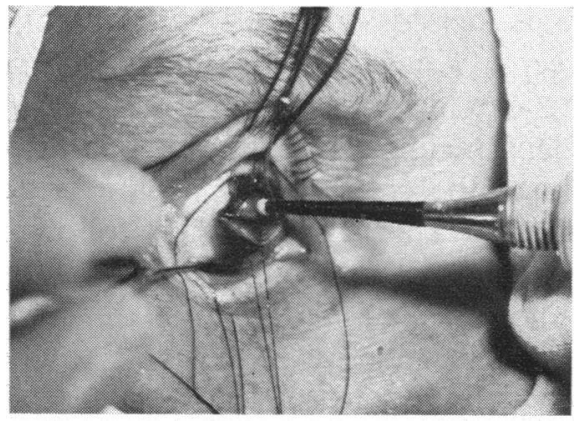

Fig. 2.-Ball-shaped end of the cryoextractor, coated with a thin layer of ice, applied to cataractous lens at 12 o'clock, near the equator.

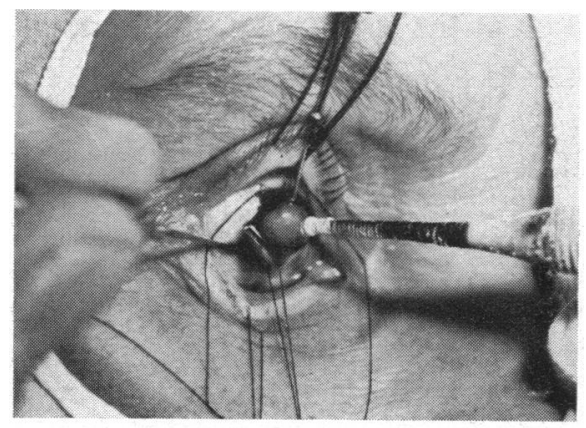

FIG. 4.-After abolition of the resistance of the zonule fibres, the cataract is delivered by sliding.

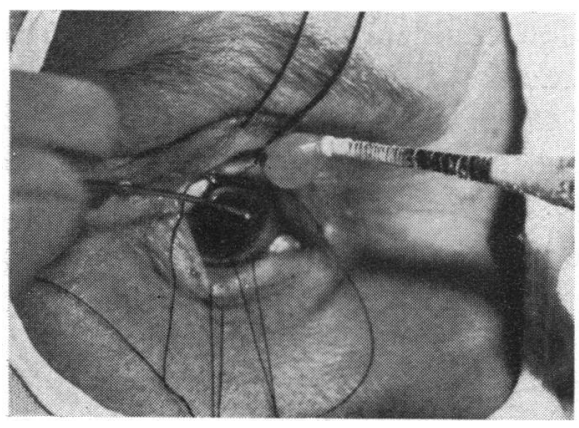

FIG. 6.-Delivered cataract adhering firmly to cryoextractor.

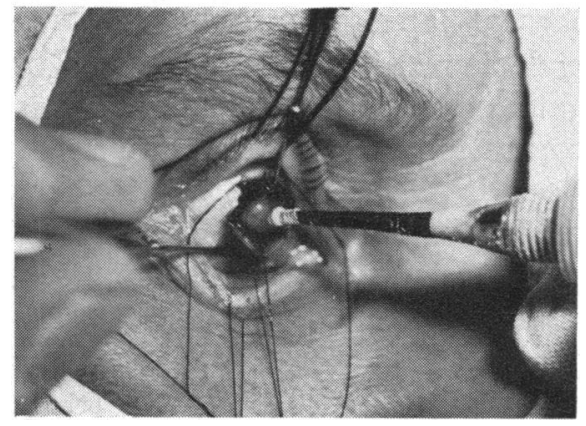

FIG. 3.-Zonule fibres broken by rotating movements.

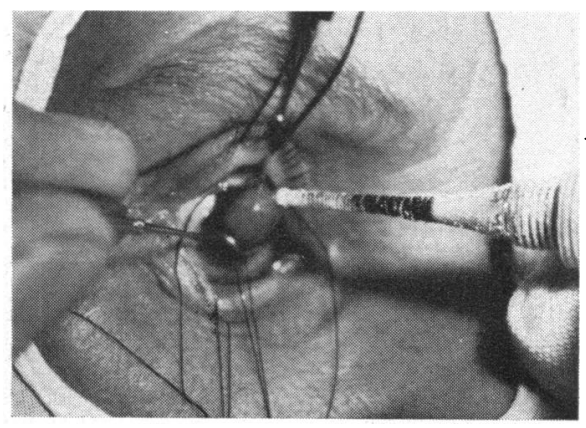

Fig. 5.-Still held by some zonule fibres, the cataract is brought into the operative wound.

This method has so far been used only in the extraction of intumescent cataracts, but it may be possible to use it for cataracts of all kinds. It must be emphasized in conclusion that the operator must be fully versed in its technicalities before applying it in practice.

Zonulolysis was not used in the present series, but a new series is in progress in which the application of low temperature is combined with zonulolysis, and the results will be published in due course. 
Summary

A new method is described of extracting intumescent cataract by means of a pencil-shaped metal instrument, named the cryoextractor. This is refrigerated in a mixture of dry ice and methyl alcohol so that when the icecoated tip is applied to the exposed lens, the capsule and the underlying cataractous masses adhere firmly to its ball-shaped end and an easy intracapsular extraction is ensured.

The results of the first fifty operations by this method are given, and the possibility of its combination with zonulysis is pointed out.

\section{REFERENCES}

Barraquer, J., and Boberg-ANs, J. (1959). Brit. J. Ophthal., 43, 69.

HRUBY, K. (1957). Klin. Mbl. Augenheilk., 130, 721.

JESS, A. (1931). Ibid., 87, 38.

LACARRÈRE J. L. (1932). Ibid., 88, 798.

LIÉGARD (1913). Ann. Oculist. (Paris), 149, 119.

OrzowskI, W. J. (1959). Klin. Oczna, 29, 349.

SMITH, H. (1926). Cited by Orłowski (1959). 